\title{
Algorithm for extracting initial and terminal contact timings during treadmill running using inertial sensors
}

\author{
Laura Prijot ${ }^{1}$, Cédric Schwartz ${ }^{1}$, Julien Watrin ${ }^{2}$, Alex Mendes ${ }^{3}$, Jean-Louis Croisier ${ }^{1}$, \\ Bénédicte Forthomme ${ }^{1}$, Vincent Denoël ${ }^{1}$, Olivier Brüls ${ }^{1}$, and Mohamed Boutaayamou ${ }^{1,4}$ \\ ${ }^{1}$ Laboratory of Human Motion Analysis, University of Liège (ULiège), Liège, Belgium \\ ${ }^{2}$ ECAM Brussels Engineering School, Brussels, Belgium \\ ${ }^{3}$ Université de Reims Champagne-Ardenne, Reims, France \\ ${ }^{4}$ INTELSIG Laboratory, Department of Electrical Engineering and Computer Science, ULiège, Liège, Belgium \\ L.Prijot@uliege.be
}

\begin{abstract}
Keywords: Running; Gait; Algorithm; Concurrent validation; Initial Contact; Terminal Contact; Temporal Events; Stride; Stance; Swing; Gyroscope; Accelerometer; IMU.

Abstract: Inertial measurement units (IMUs) are now considered as an economical solution for long term assessment in real conditions. However, their use in running gait analysis is relatively new and limited. Detecting the timing at which the foot strikes the ground (initial contact, IC) and the timing at which the foot leaves the ground (terminal contact, TC) gives access to many relevant temporal parameters such as stance, swing or stride durations. In this paper, we present an original algorithm to extract IC and TC timings and associated parameters from running data. These data have been measured using a newly developed IMU-based hardware system in ten asymptotic participants who ran at three speeds (slow, normal, and fast) with different running patterns (natural, rearfoot strike, mid-foot strike, and forefoot strike). This algorithm has been validated against a $200 \mathrm{~Hz}$ video camera based on 7056 IC and TC timings and 6861 temporal parameters. This algorithm extracted ICs and TCs with an accuracy and precision of (median [1 1 st quartile; $3^{\text {rd }}$ quartile]) $5 \mathrm{~ms}$ [-5 ms, $\left.15 \mathrm{~ms}\right]$ and $0 \mathrm{~ms}$ [-5 ms, $5 \mathrm{~ms}$, respectively. The relative errors in the extraction of stride and stance durations are $-1.56 \pm 3.00 \%$ and $0.00 \pm 1.32 \%$, respectively.
\end{abstract}

\section{INTRODUCTION}

Quantitative analysis of running is of critical interest to the sports science field. For example, this analysis can give insight into aetiology or treatment and recovery of running injuries. In the same manner, it can help sports coaches to improve the performances of their athletes. Initial contact (IC) and terminal contact (TC) are key timings in running: IC occurs at landing when the foot initiates contact with the ground while TC is when the foot ends contact. From these two key timings, it is possible to compute relevant temporal parameters, such as stance, swing or stride durations.

The stance phase, also known as the groundcontact phase, starts at the foot IC and ends at TC. The swing phase starts at TC and ends at the next IC. Finally, a stride phase is the duration between two ipsilateral ICs. Temporal parameters are related to running performances: for instance, a shorter contact time is linked to a good running economy and a faster speed (Weyand, 2000).

Traditionally, timings are detected by using force platforms. Nevertheless, these systems can only be used in controlled laboratory environments where the capture volume could be limited to a few steps.

The rapid technological advances in microelectro-mechanical systems have allowed the inertial measurement units (IMUs) to become light, small, and relatively cheap. Due to their portability and low power consumption, IMU-based systems allow obtaining real condition data.

IMUs have shown to give accurate and reliable information on walking (Boutaayamou et al., 2015 and 2016). However, running differs from walking. As the speed increases, the double support phase (both feet simultaneously touching the ground) of the walking gait cycle is replaced by a double swing phase, where both feet are in the air. Indeed, by 
definition, someone is running if both feet are never simultaneously touching the ground. Moreover, when walking, people are usually landing on their heel first. However, during running, there are three possible different landing strategies: rearfoot strike (RFS), mid-foot strike (MFS), and forefoot strike (FFS). Compared to walking, the biomechanics involved in running is also different: a wider range of motion of all the lower limb joints, higher impact forces, and higher eccentric muscle contraction (Nicola et al., 2012).

The use of IMU sensors in running gait analysis is relatively new. In the literature, different localisations for IMU sensors are considered such as trunk (Bergamini et al., 2012) or tibia (Purcell et al., 2006). Among all existing studies, only a few of them include a concurrent validation of their algorithm using a reference system. Both Chew et al. (2017) and Falbriard et al. (2018) used the signal of an IMU placed on the dorsal side of the foot to compute ICs and TCs. The first one used a threshold-based method, while the second one compared different algorithms. However, to the authors' knowledge, there is no study available using foot-worn IMU sensors that take into account the different existing landing strategies.

In this work, we present a newly developed algorithm to extract IC timing and TC timing extracted from IMU signals measured at the level of the foot (toe and heel). From these timings, the ipsilateral stance, swing, and stride durations are computed. This algorithm is tested on data obtained from ten healthy participants running at steady speeds on a treadmill. Furthermore, we validated this algorithm against synchronously recorded reference data obtained from a frame-by-frame analysis of 2D high-speed $(200 \mathrm{~Hz})$ videos.

\section{METHOD}

\subsection{Participants and Treadmill Running Setting}

In total, ten asymptotic participants ( 7 men and 3 women), who were regularly active at the time of the tests, were volunteered for this study. The set of participants includes both recreational and professional runners. They were all informed with the procedure and they have all signed an informed consent.

Table 1 shows the anthropometric characteristics
Table 1: Anthropometric characteristics of the participants measured at the time of the test

\begin{tabular}{|l|l|}
\hline & Mean \pm STD \\
\hline Age [years] & $26.1 \pm 3.9$ \\
\hline Height $[\mathrm{cm}]$ & $179.3 \pm 11.4$ \\
\hline Body mass $[\mathrm{kg}]$ & $70.0 \pm 12.3$ \\
\hline
\end{tabular}

(mean \pm standard deviation (STD)) of these participants. Among them, seven were naturally RFS while two were MFS, and one was FFS. Each participant was equipped with an IMU-based hardware system (Boutaayamou et al., 2019) integrating three-axis accelerometers (range: $\pm 16 \mathrm{~g}$ ) and three-axis gyroscopes (range: $2000 \mathrm{deg} / \mathrm{s}$ ). This system includes an acquisition box (memory, microcontroller, and battery) linked by wires to four small IMU sensors $(2.1 \times 1.0 \times 0.8 \mathrm{~cm}$, weight $=16 \mathrm{~g})$. Consequently, it is portable with an autonomy of 4h30. The IMU acquisition frequency is $200 \mathrm{~Hz}$. No restrictions on the shoes were imposed, to enlarge the range of applications of the algorithm.

The sensors were directly attached to the right shoe at the level of the first distal phalange (toe), calcaneus (heel), the fifth metatarsal, and dorsal side of the foot. In this work, only the toe and the heel sensors will be considered. The fixation procedure used has been validated in the case of walking (Boutaayamou et al., 2015) and shows satisfying results for running gait analysis.

The three-dimensional linear acceleration signals $\left[\mathrm{m} / \mathrm{s}^{2}\right]$ are denoted by $a_{x}, a_{y}$, and $a_{z}$, while the threedimensional angular velocity signals [deg/s] are denoted by $\omega_{x}, \omega_{y}$, and $\omega_{z}$ along sensitive axes represented schematically in Figure 1 .

Each test began with a standardized time to warm up and to become familiar with the treadmill and instrumentation system (during approximately five minutes). At the same time, a preferential running speed (PRS) is selected with the participant, at which he should be able to run during ten minutes without loss of intensity. The PRS (mean \pm STD) of the volunteers is $8.3 \pm 1.3 \mathrm{~km} \cdot \mathrm{h}^{-1}$. During the tests, they were asked to run at three different speeds: slow (computed by PRS- $0.25 \times \mathrm{PRS}$ ), normal (PRS), and fast (computed by PRS+0.25 $\times$ PRS). At each speed, the participants performed six trials (in the following order): three with a natural foot strike pattern, one rearfoot strike (RFS), one mid-foot strike (MFS), and one forefoot strike (FFS). In total, the participants were asked to perform 18 trials of $60 \mathrm{~s}$. The minimum total test duration was 69 minutes per participant, including 3 minutes of rest between each trial. All running tests were performed at the Laboratory of 
Human Motion Analysis (University of Liège, Belgium), on a treadmill (SportsArt T650). At the same time, all the trials were recorded using a 2D high-speed video camera (Basler Pilot) with a sampling frequency of $200 \mathrm{~Hz}$. This video camera will be used as the reference system. Signal and data processing were carried out using the software Matlab $^{\circledR}$ (R2017a, Mathworks, Natick, MA, USA).

\subsection{Extraction algorithm of IC and TC Timings}

The proposed algorithm first computes an estimated IC based on the average stride duration. Then, an exact IC timing is obtained from the different linear accelerations. Subsequently, TCs are found between two successive ICs.

The first step is to obtain an estimated average stride duration, $d_{\text {stride }}[s]$, based on the Fourier Fast Transform of the heel angular velocity signal (heel $\left.\omega_{y}\right)$. The first peak, which is also the highest, corresponds to the stride frequency $[\mathrm{Hz}] . d_{\text {stride }}$ is, then, obtained from this stride frequency using the following formula

$$
d_{\text {stride }}=\frac{1}{\text { stride frequency }} \text {. }
$$

Alternatively, $d_{\text {stride }}$ can be obtained from the auto-correlation of the same signal. In that case, the positive lag corresponding to the first positive local maxima after 0 is the average $d_{\text {stride }}$ (available in Matlab $^{\circledR}$ using the function $x$ corr).

After computing $d_{\text {stride }}$, estimated ICs are obtained in the filtered heel $\omega_{y}$ signal. The filter used is a high pass Butterworth filter of order 4 with a cut-off frequency of $15 \mathrm{~Hz}$. A high pass filter allows to remove the movement components of the signal and to keep only the shock parts. Estimated ICs can then be obtained by detecting a minimum in the filtered heel $\omega_{y}$. The distance between two successive minima is imposed to be of at least $85 \%$ of $d_{\text {stride }}$, allowing for small variations of $d_{\text {stride }}$ at each stride.

Potential exact ICs are obtained by looking for

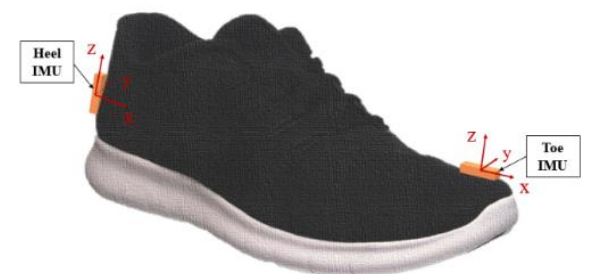

Figure 1: Schematic illustration of the position of the IMU sensors used in the proposed algorithm, including the three local axes (X-axis, Y-axis, and Z-axis). The two sensors are placed on the right shoe at the level of the first distal phalange (toe) and at the calcaneus (heel).

local extrema, in a time window around the estimated IC, in different linear acceleration signals of both sensors. Namely, the algorithm is looking for: a local minimum in toe $a_{x}$, local minimum in toe $a_{z}$, local minimum in heel $a_{x}$, and local maximum in heel $a_{z}$, in the time window [-20 ms; $5 \mathrm{~ms}]$ around the estimated IC.

Then, the exact IC corresponds to the first time instant among all these extrema. The acceleration signals in the transverse direction ( $\mathrm{Y}$-axis) are not considered since they are runner dependent. For instance, they can be influenced by foot movements like supination or pronation.

As the tip is always the last part of the foot in contact with the treadmill, TCs will be detected using the toe sensor. The toe total acceleration in the sagittal plane, given by

$$
a_{\text {sagi }}=\sqrt{a_{x}+a_{z}}
$$

has shown the highest accuracy.

TCs are determined based on the intuitive principle that there is always a TC between two successive ICs. Hence, for each stride $i$, a $T C(i)$ will be searched in the time window between $I C(i)$ and $I C(i+1)$. This window can be further reduced to increase the accuracy of the event extraction method. The upper bound of the time interval can be obtained based on the definition of running: someone is running if there is a double float phase, where both legs are in the swing phase simultaneously. This is only possible if the stance phase lasts for less than $50 \%$ of the stride duration. 


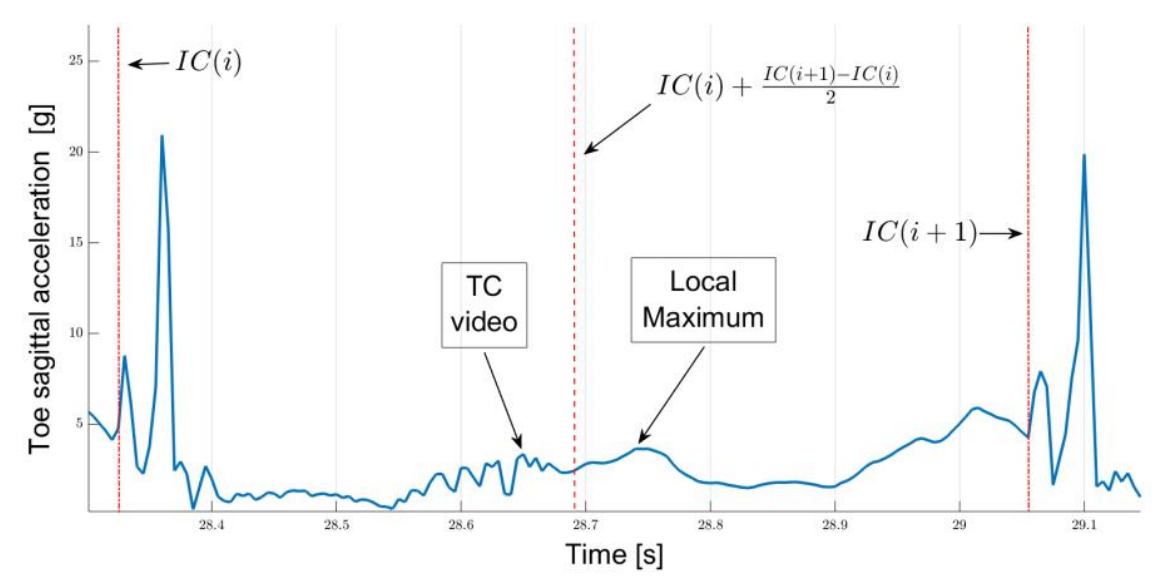

Figure 2: Determination of TCs using the toe sagittal acceleration signal. TC is found between two successive ICs. An upper limit can be obtained using the definition of running: the stance duration must be less than $50 \%$ of the stride duration. This prevents to wrongly detect local maximum coming from the movement acceleration. The signal flat phase can be used as a lower limit, which can be detected using the entropy of the signal over a sliding window.
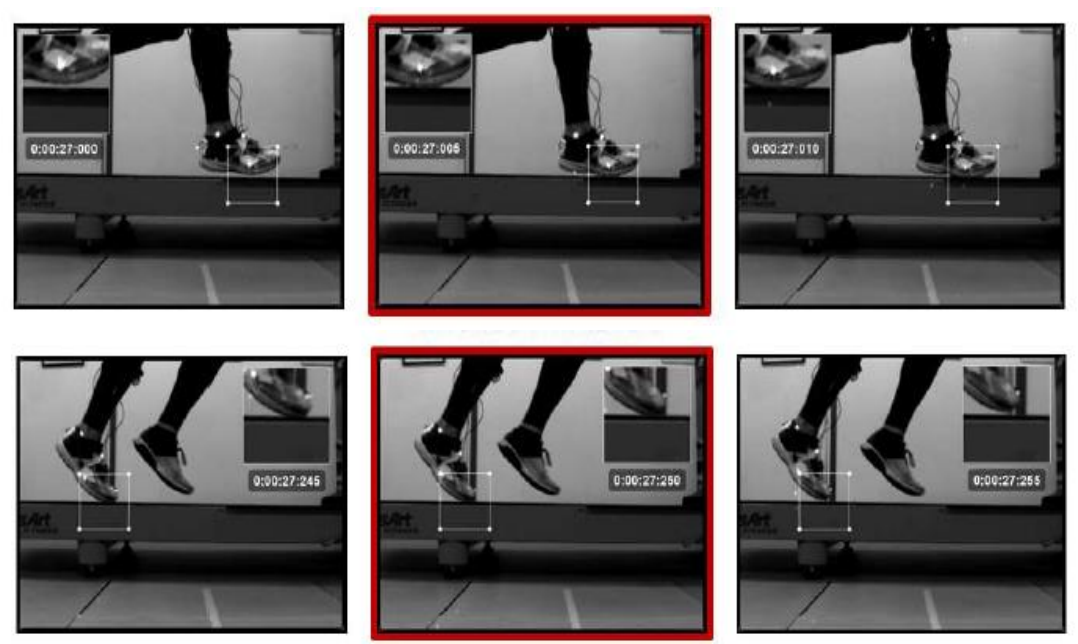

Figure 3: Determination of IC and TC timings using the 2D high-speed video camera. IC (upper pictures) is the first frame where the pixels representing one shoe are directly in contact with those representing the belt of the treadmill. TC (lower pictures) corresponds to the last frame where the pixels of the shoe are in contact with those of the treadmill.

Hence, the upper limit is defined as follows

$$
\operatorname{Lim}_{\text {sup }}=I C(i)+\frac{I C(i+1)-I C(i)}{2} \text {. }
$$

This limits the application of the algorithm to only running cases. However, this improves the accuracy. In fact, in some cases, the acceleration linked to the swing movement of the foot is higher than the shock corresponding to the TC (see Figure 2).

Furthermore, the lower bound of the time window $\left(\operatorname{Lim}_{\text {inf }}\right)$ is obtained using the entropy of the signal. During the stance phase, the foot has a constant acceleration and this signal flat zone is characterized by a low entropy. Hence, the lower limit is obtained by computing the entropy over a sliding window. The size of the window has been determined empirically: on one side, it should be as small as possible to have good local information. On the other side, it must be large enough to not detect the flatter zone that appears for some runners after the toe-off peak. This was generally a problem for FFS running patterns. A window of 15 samples (i.e., $75 \mathrm{~ms}$ ) has shown good results for all participants.

$T C(i)$ is then determined by finding a maximum in the toe sagittal acceleration signal over the time window : $\left[\operatorname{Lim}_{\text {inf }}(i) ; \operatorname{Lim}_{\text {sup }}(i)\right]$.

\subsection{Concurrent Validation and Evaluation Methods}

The reference timings are obtained from a frame-byframe analysis of 2D high speed videos. A precise definition is used to select the frame corresponding 
to an IC and to a TC: IC is the first frame where the pixels representing one shoe are directly in contact with those representing the belt of the treadmill. In other words, it is the first frame where there are no white pixel (i.e., background pixel) in-between the shoe and the treadmill.

Conversely, TC corresponds to the last frame where the pixels of the shoe are in contact with those of the treadmill (see Figure 3).

Finally, the different temporal parameters are computed from IC and TC timings, as follows

$$
\begin{gathered}
d_{\text {stance }}(i)=T C(i)-I C(i), \\
d_{\text {swing }}(i)=I C(i+1)-T C(i), \\
d_{\text {stride }}(i)=I C(i+1)-I C(i) .
\end{gathered}
$$

The reference system has a maximum achievable resolution of $5 \mathrm{~ms}$. Additionally, at some point in the video, there are two identical frames following each other. In that case, a $5 \mathrm{~ms}$ error can also occur.

These reference timings are used to concurrently validate the events obtained using the proposed algorithm. For each stride, the results for (1) IC, (2) TC, (3) $d_{\text {stance }}$, and (4) $d_{\text {stride }}$ are computed. The results for $d_{\text {swing }}$ have been computed but are not shown in this paper

Finally, the accuracy and precision of ICs and TCs extraction is quantified by the mean and STD or median and inter-quartile range (IQR) values (i.e., $1^{\text {st }}$ quartile $\left(\mathrm{Q}_{1}\right)$; $3^{\text {rd }}$ quartile $\left.\left(\mathrm{Q}_{3}\right)\right)$ of the differences between these timings and the reference system, depending on the normality of data distributions. This is done for each participant separately and for all participants together. The normality of data distributions is tested using Jarque-Bera test (available in Matlab ${ }^{\circledR}$ using the function jbtest). Additionally, relative errors are computed as the mean of the strideby-stride differences between the IMU temporal parameter and the reference temporal parameter divided by the reference temporal parameter. These errors are only meaningful for temporal parameters and they will not be computed for timings.

\section{RESULTS}

This work focuses on running trials from the acquired data. In some trials, particularly at low speeds, some participants exhibited a double support phase. Consequently, as these trials are considered as walking trials, they have been excluded from this study. In total, 39 trials out of 183 were not considered.
Table 2: Intra-participant differences between IMU timings/temporal parameters and reference data. It includes the median and the interquartile range values ([ $1^{\text {st }}$

\begin{tabular}{|c|c|c|c|}
\hline $\begin{array}{l}\text { Partici- } \\
\text { pants }\end{array}$ & $\begin{array}{l}\text { Running } \\
\text { timings/ } \\
\text { parameters }\end{array}$ & $\begin{array}{c}\text { Median } \\
{\left[\mathrm{Q}_{1} ; \mathrm{Q}_{3}\right]} \\
{[\mathrm{ms}]}\end{array}$ & $\begin{array}{l}\text { Nbr. } \\
\text { of } \\
\text { obs. }\end{array}$ \\
\hline 1 & $\begin{array}{l}\mathrm{IC} \\
\mathrm{TC} \\
d_{\text {stance }} \\
d_{\text {stride }} \\
\end{array}$ & $\begin{array}{l}0[-10 ; 10] \\
0[-5 ; 5] \\
5[-5 ; 15] \\
0[-10 ; 10] \\
\end{array}$ & $\begin{array}{l}211 \\
205 \\
205 \\
191 \\
\end{array}$ \\
\hline 2 & $\begin{array}{l}\mathrm{IC} \\
\mathrm{TC} \\
d_{\text {stance }} \\
d_{\text {stride }}\end{array}$ & $\begin{array}{c}-10[-20 ; 0] \\
0[-5 ; 5] \\
10[-5 ; 25] \\
0[-10 ; 10]\end{array}$ & $\begin{array}{l}306 \\
306 \\
306 \\
291\end{array}$ \\
\hline 3 & $\begin{array}{l}\mathrm{IC} \\
\mathrm{TC} \\
d_{\text {stance }} \\
d_{\text {stride }}\end{array}$ & $\begin{array}{l}0[-20 ; 20] \\
0[-5 ; 5] \\
5[-15 ; 25] \\
0[-10 ; 10]\end{array}$ & $\begin{array}{l}437 \\
437 \\
437 \\
403\end{array}$ \\
\hline 4 & $\begin{array}{l}\mathrm{IC} \\
\mathrm{TC} \\
d_{\text {stance }} \\
d_{\text {stride }}\end{array}$ & $\begin{array}{c}5[0 ; 10] \\
0[0 ; 10] \\
-5[-10 ; 0] \\
0[-10 ; 10]\end{array}$ & $\begin{array}{l}366 \\
366 \\
365 \\
348 \\
\end{array}$ \\
\hline 5 & $\begin{array}{l}\mathrm{IC} \\
\mathrm{TC} \\
d_{\text {stance }} \\
d_{\text {stride }}\end{array}$ & $\begin{array}{c}10[5 ; 15] \\
0[-5 ; 5] \\
-10[-15 ;-5] \\
0[-10 ; 10]\end{array}$ & $\begin{array}{l}484 \\
394 \\
385 \\
462\end{array}$ \\
\hline 6 & $\begin{array}{l}\mathrm{IC} \\
\mathrm{TC} \\
d_{\text {stance }} \\
d_{\text {stride }}\end{array}$ & $\begin{array}{c}10[5 ; 15] \\
0[-5 ; 5] \\
-10[-15 ;-5] \\
0[-5 ; 5]\end{array}$ & $\begin{array}{l}332 \\
332 \\
332 \\
314\end{array}$ \\
\hline 7 & $\begin{array}{l}\mathrm{IC} \\
\mathrm{TC} \\
d_{\text {stance }} \\
d_{\text {stride }}\end{array}$ & $\begin{array}{c}15[5 ; 25] \\
0[-5 ; 5] \\
-15[-30 ; 0] \\
0[-10 ; 10]\end{array}$ & $\begin{array}{l}129 \\
129 \\
129 \\
125\end{array}$ \\
\hline 8 & $\begin{array}{l}\mathrm{IC} \\
\mathrm{TC} \\
d_{\text {stance }} \\
d_{\text {stride }}\end{array}$ & $\begin{array}{l}0[-15 ; 15] \\
0[-5 ; 5] \\
0[-20 ; 20] \\
0[-15 ; 15]\end{array}$ & $\begin{array}{l}312 \\
213 \\
213 \\
303\end{array}$ \\
\hline 9 & $\begin{array}{l}\mathrm{IC} \\
\mathrm{TC} \\
d_{\text {stance }} \\
d_{\text {stride }} \\
\end{array}$ & $\begin{array}{c}10[5 ; 15] \\
0[-5 ; 5] \\
-5[-15 ; 5] \\
0[-10 ; 10]\end{array}$ & $\begin{array}{l}468 \\
450 \\
448 \\
451 \\
\end{array}$ \\
\hline 10 & $\begin{array}{l}\mathrm{IC} \\
\mathrm{TC} \\
d_{\text {stance }} \\
d_{\text {stride }} \\
\end{array}$ & $\begin{array}{l}5[-15 ; 25] \\
0[-5 ; 5] \\
-5[-25 ; 15] \\
0[-10 ; 10]\end{array}$ & $\begin{array}{l}608 \\
571 \\
567 \\
586 \\
\end{array}$ \\
\hline
\end{tabular}
quartile $\mathrm{Q}_{1} ; 3^{\text {rd }}$ quartile $\left.\mathrm{Q}_{3}\right]$ ) as well as the number of observations (nbr. of obs.). 
Additionally, some trials have been reclassified according to the real running pattern observed that, in some cases, was different from the supposed running pattern. Indeed, some participants had difficulties in voluntarily performing MFS or FFS.

First of all, an intra-participant comparison between the IMU results and reference results is carried out. In this paper, the median is used as the data are not normality distributed. However, in general in this study, the mean and median values and STD values IQR ones were similar. The same conclusion can be drawn for STD values IQR ones.

Table 2 summarizes the results for each participant, the values have been rounded to the sample period (i.e., $5 \mathrm{~ms}$ ) of the hardware systems. This analysis includes all the valid trials (different speeds and different foot strikes) and at least 30 valid strides per trial, when available. The number of observations depends on the number of valid events taken into account. In the case of ICs, the mean of the extraction accuracies is $5 \mathrm{~ms}$. Consequently, the algorithm tends to detect the ICs one frame later than the reference system. The mean of the extraction precisions obtained in the case of IC is 10 $\mathrm{ms}$. The worst-case for the IC determination appears for participants 3 and 10, with an IQR of $20 \mathrm{~ms}$ away from the median value. The best case is for participant 4 with a median error of one sample (i.e., $5 \mathrm{~ms}$ ) and IQR of $5 \mathrm{~ms}$ around this median error. In that case, the precision obtained exactly corresponds to the maximum achievable precision. Indeed, the maximum precision depends on the sampling rate of both the IMU system and the high-speed video, as well as the $5 \mathrm{~ms}$ error than can be explained by errors in the reference system.

In the case of TCs, the results obtained with IMU are similar to those obtained with the reference system. Indeed, the mean of median errors between the two systems is $0 \mathrm{~ms}$ and the mean of the IQRs is $5 \mathrm{~ms}$, for all participants. Therefore, the algorithm can detect TCs with the maximum possible accuracy.

For the stance duration, the algorithm tends to underestimate the duration compared to the reference values. This can be explained by the fact that ICs are generally detected later with the algorithm. The mean of the median values is $-3 \mathrm{~ms}$, which is less than one sample of difference and less than the maximum accuracy. The mean of the variability values is $12.5 \mathrm{~ms}$, this is slightly higher than the maximum precision expected. Indeed, $I C(i)$ can be determined with a maximum precision of $5 \mathrm{~ms}$ and $T C(i)$ can also be determined with a maximum
Table 3: Inter-participant comparison including the extraction accuracies and precisions of 7 participants, running with their preferential running style at speeds ranging from 7.1 to $9 \mathrm{~km} \cdot \mathrm{h}^{-1}$.

\begin{tabular}{|l|c|c|c|}
\hline $\begin{array}{l}\text { Running } \\
\text { timings/ } \\
\text { parameters }\end{array}$ & $\begin{array}{c}\text { Mean } \pm \\
\mathrm{STD} \\
{[\mathrm{ms}]}\end{array}$ & $\begin{array}{c}\text { Median } \\
{\left[\mathrm{Q}_{1} ; \mathrm{Q}_{3}\right]} \\
{[\mathrm{ms}]}\end{array}$ & $\begin{array}{c}\text { Median } \\
{\left[\mathrm{Q}_{1} ; \mathrm{Q}_{3}\right]} \\
{[\%]}\end{array}$ \\
\hline $\mathrm{IC}$ & $5 \pm 9$ & $5[-5 ; 15]$ & $/$ \\
\hline $\mathrm{TC}$ & $1 \pm 4$ & $0[-5 ; 5]$ & $/$ \\
\hline$d_{\text {stance }}$ & $-5 \pm 15$ & $-5[-15 ; 5]$ & $\begin{array}{c}-1.56 \\
{[-4.56 ; 2.56]}\end{array}$ \\
\hline$d_{\text {Stride }}$ & $0 \pm 10$ & $0[-10 ; 10]$ & $\begin{array}{c}0.00 \\
{[-1.32 ; 1.32]}\end{array}$ \\
\hline
\end{tabular}

precision of $5 \mathrm{~ms}$. As the errors may cumulate, a maximum precision of $10 \mathrm{~ms}$ is expected for durations. However, the variability is of $5 \mathrm{~ms}$ for three participants out of ten.

The accuracy for the stride duration is $0 \mathrm{~ms}$ for all participants, which is the best possible achievable accuracy. The precision obtained is on average $10 \mathrm{~ms}$, which is the expected precision, as explained before. Note that, one participant out of the ten has a better precision $(5 \mathrm{~ms})$ and only one participant has a worst precision $(15 \mathrm{~ms})$ than the one expected.

Finally, an inter-participant comparison is done, including only the natural foot strike trials at PRS condition (i.e., three trials per participant). We did not include the trials performed by three participants as at least one of the three above mentioned trials was not valid. The speed of the trials considered was between 7.1 and $9.0 \mathrm{~km} \cdot \mathrm{h}^{-1}$.

Table 3 provides the mean \pm STD as well as the median and IQR values of the differences between the extracted IMU values and the reference values. The mean errors and the median errors are similar for all timings and temporal parameters. The extraction precisions expressed in terms of STD are identical to those expressed in terms of IQR except for the stance duration, where the STD value is influenced by some outlier values.

The extraction accuracy in the case of ICs is $5 \mathrm{~ms}$. The algorithm tends, then, to detected ICs one sample later than those extracted by the reference system. This could be explained by the fact that IMUs will detect the interaction (shock) between the shoe and the belt of the treadmill while, in the video, the selected frame is the one when the shoe and the treadmill touch each other but have not yet interacted. The precision obtained for the ICs is $10 \mathrm{~ms}$, which is one frame higher than the maximum achievable 
precision. On the other side, TCs are extracted with both the maximum achievable accuracy (i.e., $0 \mathrm{~ms}$ ) and precision (i.e., $5 \mathrm{~ms}$ ).

The stance durations tend to be underestimated by the algorithm. On average, they are $5 \mathrm{~ms}$ shorter than those obtained with the reference system. Again, this is explained by the fact that ICs have a tendency to be detected $5 \mathrm{~ms}$ later with the IMUs. Finally, the stride duration, which only depends on successive ICs, are extracted with the best possible accuracy (i.e., $0 \mathrm{~ms}$ ) and a precision equal to the maximum expected precision due to the accumulation of errors. Indeed, there could be $5 \mathrm{~ms}$ of error in the first IC $(I C(i))$ and $5 \mathrm{~ms}$ of error for the successive IC $(\operatorname{IC}(i+1))$. All in all, it can be seen that the inter-participants and intraparticipant comparison give similar results.

It is also interesting to express the errors in both stance and stride duration estimates as a percentage of the total duration. The $d_{\text {stance }}$ relative error is (median $\left.\left[\mathrm{Q}_{1}, \mathrm{Q}_{3}\right]\right)-1.56 \%[-4.56 \% ; 2.56 \%]$ and the maximum relative error is $-9.52 \%$. The $d_{\text {stride }}$ relative error is (median $\left.\left[\mathrm{Q}_{1}, \mathrm{Q}_{3}\right]\right): 0.00 \%[-1.32 \% ; 1.32 \%]$ and the maximum computed error is $4.49 \%$.

\section{DISCUSSION}

This article presents an original algorithm to extract the two main timings (ICs and TCs) at different running speeds (slow, normal, and fast) and with different running styles (natural, RFS, MFS, and FFS). The data collected for this work are obtained using two IMU sensors placed on regular shoes at the level of the heel (calcaneus) and toe (first distal phalange).

Only the right shoe has been used in this work. However, the algorithm is supposed to work in the same way for the left foot. Additionally, the IMU hardware system used here can record the data of up to four sensors at the same time. It is thus possible to record the data of both legs simultaneously. Therefore, it would be possible to obtain other parameters, such as the step duration. Besides, it would be possible to make a comparison between the two legs, which has a wide range of applications, including monitoring recovery after injury or surgery.

The performance of the algorithm is determined by a concurrent validation with $2 \mathrm{D}$ high-speed videos, recorded simultaneously. The algorithm presented here has been concurrently validated using a total of 7056 timings and 6861 temporal parameters. This comparison has shown a good agreement between timings obtained using the IMU signals and timings detected on the $2 \mathrm{D}$ videos. The measures include running speeds ranging from 6.0 to $11.3 \mathrm{~km} . \mathrm{h}^{-1}$. The obtained global extraction accuracy and precision (median $\left[\mathrm{Q}_{1} ; \mathrm{Q}_{3}\right]$ ) is $5 \mathrm{~ms}[-5 \mathrm{~ms} ; 15$ $\mathrm{ms}]$ and $0 \mathrm{~ms}$ [ $-5 \mathrm{~ms} ; 5 \mathrm{~ms}$ for, respectively, ICs and TCs. Besides, the accuracy and precision for the stance durations and stride durations (median $\left[\mathrm{Q}_{1}\right.$; $\left.\left.\mathrm{Q}_{3}\right]\right)$ are $-5 \mathrm{~ms}[-15 \mathrm{~ms} ; 5 \mathrm{~ms}]$ and $0 \mathrm{~ms}$ [-10 ms; 10 $\mathrm{ms}]$, respectively. This corresponds to a relative error of respectively $-1.56 \pm 3.00 \%$ and $0.00 \pm 1.32 \%$.

The stride duration average error obtained here (i.e., $0 \mathrm{~ms}$ ) is consistent with the one measured by Chew et al. (2018), which is between $-0.44 \mathrm{~ms}$ and $0.33 \mathrm{~ms}$. However, Chew et al. (2018) used an algorithm based on a thresholding-method that relies on experimental values needed to determine the threshold. This is not the case for the algorithm presented here. Similarly, the stance duration errors are similar to those found by Purcell et al. (2006). They found an error (mean \pm STD) of $0 \pm 12 \mathrm{~ms}$ and $-2 \pm 3 \mathrm{~ms}$, depending on the running speed, using a tibial accelerometer. However, they used a force platform with higher accuracy than the $2 \mathrm{D}$ video system used here. Falbriard et al. (2018) found better accuracy and precision (median $\left[\mathrm{Q}_{1}, \mathrm{Q}_{3}\right]$ ): 2 [1 ms, 3 $\mathrm{ms}$ ] for IC and only a better precision for TC (4 ms [2 $\mathrm{ms}, 6 \mathrm{~ms}])$. Nevertheless, this precision cannot be achieved here with the $200 \mathrm{~Hz}$ reference system used.

The algorithm presented here is only valid for steady state running over a treadmill. Walking cases cannot be analysed using the present method, however, there exist algorithms to detect the type of activity (walking, running, and rest). Once the activity is appropriately determined, either a walking or a running algorithm can be selected to extract temporal events.

\section{CONCLUSION}

In this article, we presented an original algorithm to extract timings (IC and TC) in the case of steadystate running over a treadmill, using IMU sensors. From these two timings, three temporal parameters can also be computed: stance, swing, and stride durations. The method developed here has the following advantages:

- The sensors are placed on the shoes and not directly on the feet, which allows running in many different conditions.

- The algorithm only uses two IMU sensors per foot: one at the level of the heel and the other at the level of the first distal phalange (toe). Additionally, only one sensor (i.e., toe sensor) is used to determine TCs with the maximum achievable precision and accuracy. 
- This method has been concurrently validated using a 2D high-speed video camera as the reference system.

- The analysis is done over a large number of strikes including a wide range of running speeds (from $6 \mathrm{~km} \cdot \mathrm{h}^{-1}$ to $11.3 \mathrm{~km} \cdot \mathrm{h}^{-1}$ ) and different running styles (natural, RFS, MFS, and FFS).

The results showed that it is possible to achieve acceptable accuracy and precision using a foot-worn IMU-based system. These results are encouraging for the use of IMU for daily and out-of-lab monitoring.

They can be seen as a good trade-off between expensive and laboratory-limited measurement instruments like force platforms that show high accuracy and wearable systems that can be found in smartwatches or in smartphones.

Future researches may focus on the use of a single IMU sensor to extract the timings and associated temporal parameters or on the detection of spatial parameters like the stride length. Further work could also focus on extracting the durations of the stride sub-phases.

\section{ACKNOWLEDGEMENTS}

The authors would like to thank all the participants who were volunteers for this study. We would also like to thank the reviewers for their relevant comments, which will be taken into consideration in future articles.

\section{REFERENCES}

Amini, N., Vahdatpour, A., Wenyao Xu, and Sarrafzadeh., M., 2011. Accelerometer-based on-body sensor localization for health and medical monitoring applications. Pervasive Mobile Computing, 7:746-760

Bergamini, E., Picerno, P., Pillet, H., Natta, F., Thoreux, P., and Camomilla, V., 2012. Estimation of temporal parameters during sprint running using a trunkmounted inertial measurement unit. Journal of Biomechanics, 45:1123-1126.

Benson, L., Clermont, C., Bošnjak, E., and Ferber, R., 2018. The use of wearable devices for walking and running gait analysis outside of the lab: A systematic review. Gait \& Posture, 63.

Boutaayamou, M., Denoël, V., Brüls, O.,Demonceau, M., Maquet, D., Forthomme, B., Croisier, J.-L., Schwartz, C., Verly, J., and Garraux. G., 2016. Extraction of temporal gait parameters using a reduced number of wearable accelerometers.

Boutaayamou, M., Denoël, V., Brüls, O., Maquet, D., Forthomme, B., Croisier, J.-L., Schwartz, C., Verly, J.,
Macq, B., Garraux, G., and Stamatakis, J., 2015. Development and validation of an accelerometerbased method for quantifying gait events. Medical Engineering and Physics, 37:226-232

Boutaayamou, M., Schwartz, C., Joris, L., Forthomme, B., Denoël, V., Croisier, J.-L., Verly, J. \& Garraux, G., and Brüls, O., 2019. Adaptive Method for Detecting Zero-Velocity Regions to Quantify Stride-to-Stride Spatial Gait Parameters using Inertial Sensors. 12th International Conference on Bio-inspired Systems and Signal Processing, 229-236.

Chew, D.-K., Ngoh, K., Gouwanda, D., and Gopalai., A., 2017. Estimating running spatial and temporal parameters using an inertial sensor. Sports Engineering, 21(2):115-122.

Falbriard, F., Meyer, B., Mariani, G., Millet, P., and Aminian, K., 2018. Accurate estimation of running temporal parameters using foot-worn inertial sensors. Frontiers in Physiology.

Hanley, B., Bissas, A., and Drake, A., 2015. The contribution of the flight phase in elite race walking. 33rd International Conference on Biomechanics in Sports, At Poitiers, France

Koska, D., Gaudel, J., Hein, T., and Maiwald, C., 2018. Validation of an inertial measurement unit for the quantification of rearfoot kinematics during running. Gait \& Posture, 64:135-140.

Maiwald, C., Sterzing, T., Mayer, T.A., and Milani, T.L., 2009. Detecting foot-to-ground contact from kinematic data in running. Footwear Science, 1(2):111-118.

Mariani, B., Rouhani, H., Crevoisier, X., and Aminian, K., 2013. Quantitative estimation of foot-flat and stance phase of gait using foot-worn inertial sensors. Gait and Posture, 37(2):229 - 234.

Nicola, T. and Jewison, D., 2012. The anatomy and biomechanics of running. Clinics in Sports Medicine, 31:187-201.

Nicolai, L., Mifsud, N., Kristensen, H., Villumsen, M., Hansen, J., and Kersting, U., 2014. Portable inertial motion unit for continuous assessment of in-shoe foot movement. Procedia Engineering, 72:208-213.

Purcell, B., Channells, J., James, D., and Barrett, R., 2006 Use of accelerometers for detecting foot-ground contact time during running. Proc. SPIE

Weyand, P.G., Sternlight,, D. B., Bellizzi, M. J., and Wright, S., 2000. Faster top running speeds are achieved with greater ground forces not more rapid leg movements. Journal of Applied Physiology, 89(5):1991-1999.

Wixted, A., Thiel, D., Hahn, A., Gore, C., Pyne, D., and James, D., 2007. Measurement of energy expenditure in elite athletes using mems-based triaxial accelerometers. Sensors Journal, IEEE, 7:481-488.

Yang, S., Mohr, C., and Li, Q., 2011. Ambulatory running speed estimation using an inertial sensor. Gait and Posture, 34(4):462 - 466. 